\title{
Evaluation of the optic nerve using strain and shear wave elastography in patients with multiple sclerosis and healthy subjects
}

\author{
Mikail İnal', Sinan Tan', M. Erhan Yumusak², Mehmet Hamdi Şahan', Murat Alpua ${ }^{3}$, \\ Kemal Örnek ${ }^{2}$
}

${ }^{1}$ Department of Radiology, ${ }^{2}$ Department of Ophtalmology, ${ }^{3}$ Department of Neurology, Kirikkale University Faculty of Medicine, Kirikkale, Turkey

\begin{abstract}
Aims: Our aim was to evaluate the elasticity features of the optic nerve using strain (SE) and shear wave elastography (SWE) in multiple sclerosis (MS) patients in comparison with healthy subjects. Material and methods: One hundred and seven optic nerves from 54 MS patients and 118 optic nerves from 59 healthy subjects were examined prospectively by SE and SWE. Optic nerves were divided into three types in accordance to the elasticity designs, as follows: type 1 predominantly blue (hardest tissue); type 2 predominantly blue/green (hard tissue); and type 3 predominantly green (intermediate tissue). Quantitative measurements of optic nerve hardness with SWE were analyzed in kilopascals. Results: Elastographic images from healthy volunteers showed mostly type 3 optic nerves $(61.9 \%)$; type 2 was also found (38.1\%), but type 1 was not observed. Elastographic examination of MS patients showed mostly type 2 optic nerves (88\%), while some type 1 (4.6\%) and type 3 optic nerves $(6.5 \%)$ were rarely observed. There was a statistically significant difference in terms of elasticity patterns between patients and healthy volunteers $(\mathrm{p}<0.001)$. Statistically significant differences were observed between patients and healthy volunteers in the analysis of SWE values $(10.381 \pm 3.48 \mathrm{kPa}$ and $33.87 \pm 11.64 \mathrm{p}<0.001)$. The receiver operating characteristic curve analysis was perfect $(0.993 ; 95 \%$ confidence interval $[\mathrm{CI}]=0.971-0.999)$, and a cut-off value of $18.3 \mathrm{kPa}$ shear had very high sensitivity and specificity for the patient group. No significant differences were observed between patients with and without previous optic neuritis. Conclusion: SE and SWE examination findings concerning the optic nerve in MS patients demonstrated remarkable differences according to the healthy group.
\end{abstract}

Keywords: elastography; shear wave; optic nerve; multiple sclerosis.

\section{Introduction}

Multiple sclerosis (MS) is a chronic inflammatory neurodegenerative disease that affects the central nervous system [1,2]. Demyelination and iron-related abnormalities are the accepted underlying pathological mechanisms $[3,4]$. Impaired vision is one of the most common symptoms of MS, and the visual system can be affected in all anatomical sites. Of these sites, the optic nerve is

Received 15.06.2016 Accepted 10.09.2016

Med Ultrason

2017, Vol. 19, No 1, 39-44

Corresponding author: Sinan Tan, MD

Kirikkale Universitesi Tip Fakültesi, Radyoloj AD,

Yenisehir Mah., Tahsin Duru Cad., No: 14, Yahsihan / Kirikkale / Turkey

Phone: +90 4404071-5407 Fax: +90 3182444697

E-mail: drsinantan@gmail.com the best described, because inflammatory demyelinating optic neuritis may be the initial presenting feature in patients with MS $[5,6]$. Conventional and non-conventional magnetic resonance imaging (MRI) techniques, such as diffusion tensor imaging, diffusion-weighted imaging, and magnetization transfer imaging are used for the diagnosis of optic neuritis [7-11]. However, MRI examinations are time consuming, expensive, less available and often require the use of gadolinium contrast.

Sonoelastography is a non-invasive ultrasound method for evaluating the elastic properties of tissues based on static compression and cross-correlation methods qualitatively and/or quantitatively. The main elastographic techniques include strain elastography (SE), which compresses the tissues axially, and shear wave elastography (SWE), which uses waves that are generated by transducers and interact with the tissue. In some studies, it has been reported that there is a connection between the 
tissue elasticity and histological features. Elastography is used successfully in prostate, lymph node, breast, thyroid, testis, renal and liver disease examination [12-16]. However, to the best of our knowledge, there has been no work in the previous literature that has investigated the optic nerve with elastography in MS patients.

The purpose of this study is to describe the elasticity features of the optic nerve using SE and SWE in MS patients with or without optic neuritis and to compare the results with those of healthy volunteers, in order to establish if these elasticity characteristics can be used to assist in the diagnosis of optic nerve involvement in MS.

\section{Material and methods}

\section{Participants}

The study was approved by the hospital ethics committee and was conducted according to the Helsinki Declaration. Voluntary informed consent was obtained from all patients included in the study.

The patients were examined in the relevant department in terms of neurological and ophthalmological pathology. The MS diagnosis was established using the revised McDonald criteria [17], and patients with relapsing-remitting MS were included in the study. None of our patients had acute optic neuritis. Previous optic neuritis was diagnosed based on typical history and clinical findings by an ophthalmologist.

Patients with systemic diseases affecting the eye, such as diabetes mellitus, Behçet's disease, and systemic hypertension were excluded. Patients with a history of ophthalmic surgical intervention, accompanying glaucoma, or advanced retinal or macular disease were also excluded.

\section{Equipment and scanning}

Sonoelastography examinations of all subjects were carried out by one of two radiologists (M.I. and S.T., 1510 years of experience with conventional sonography and 1-5 years of experience within elastography, respectively) with the patient in the supine position using LOGIQ E9 sonographic system (GE Healthcare) with elastography software and a linear array probe of 6-9 MHz. The optic nerve sheath caliber was measured $3 \mathrm{~mm}$ behind the globe between the external hypoechogenic borders using an axis perpendicular to the optic nerve with the eyes keep in nearly a neutral state in supine position $[18,19]$.

A coupling gel was applied to the eyelid to acquire images, and subjects were instructed to keep their eyes closed and refrain from moving during the investigation. The B-mode and elastographic images were shown simultaneously side by side on a split-screen display. Images in SE were performed by applying mild compression of the transducer with the hand-held device. A compression bar indicator of 1 to 7 was monitored in real time on the screen, and images were analyzed when the ideal compression was applied, which was in the 5-7 bar range. Elastography images according to the different levels of strain were displayed on the picture over a grayscale ground in a color diagram that included red (softest component), green (intermediate stiffness) and blue (hardest component) [14].

Each patient was examined without compression using SWE, and the images were saved in the transverse plane from inside the equator of the eyeball. Both static images and video clips (at least 5 seconds per case) were saved digitally on the machine to analyze subsequently.

\section{Data analysis}

Each case used to assess the optic nerve elasticity pattern in SE was reviewed by visual examination of the movement video series, and the final interpretations were determined by two radiologists through consensus. The color mapping of the optic nerve strain was divided into three main types (type 1: predominantly blue - hardest tissue; type 2: predominantly blue/green - hard tissue; type 3: predominantly green - intermediate tissue) (fig 1).

Quantitative analysis of optic nerve hardness with SWE used kilopascal $(\mathrm{kPa})$ measurement with a color

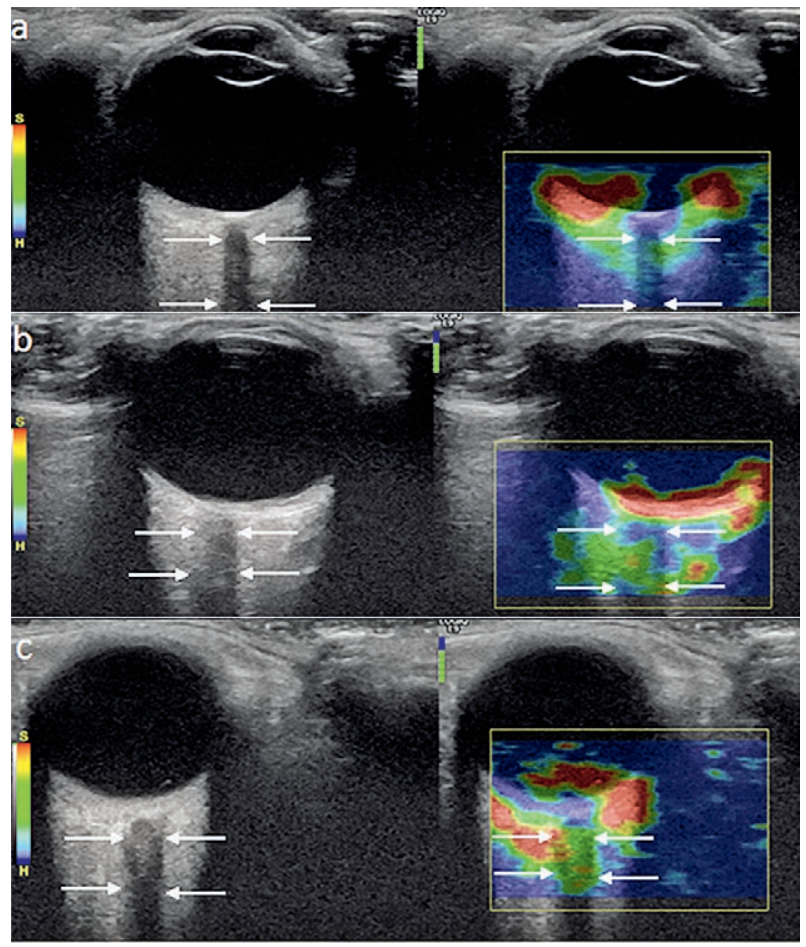

Fig 1. Grayscale (left) and color (right) sonograms representing different elastographic patterns of the optic nerve (arrows): a) Type 1 , in a 34 -year-old woman with multiple sclerosis; b) type 2 , in a 53-year-old woman with multiple sclerosis; c) type 3 , in a 37 -year-old woman with multiple sclerosis. 
scale of $0-150 \mathrm{kPa}$ with a circular region of interest (ROI) area in the interval of $2-4 \mathrm{~mm}$, as displayed in figure 2. The quantitative value for each optic nerve was obtained with at least three measurements and the mean values were used for the statistical analysis.

\section{Statistical analysis}

We used the SPSS software package program (SPSS, Chicago, IL, USA), version 20.0, for the statistical analysis. Values are expressed as mean \pm standard deviation (SD). The male-to-female ratio of the groups was compared using the Chi-square test. We assessed the dissimilarities between genders and the dispersion of groups using the one-way analysis of variance (ANOVA) test. We classified $p$-values less than 0.05 as statistically significant.

Elasticity designs were investigated using the Chisquare test to assess the relationships between them, and we classified primary color designs and color designs to appraise the diagnostic ability of SE. Non-parametric tests were used in the assessment of SWE values and the optic nerve diameter. The independent Student's t-test (unpaired) was applied in the analysis of the SWE values and the relationship between age and the optic nerve diameter. The Pearson correlation test was used to evaluate the connection between disease duration and SWE values. Confidence intervals (CIs) were used to clarify the correctness of the diagnostic factors (sensitivity, specificity, positive predictive value $[\mathrm{PPV}]$, and negative predictive value $[\mathrm{NPV}]$ ) for SWE values. To evaluate the diagnostic importance of SWE values, receiver operating characteristic (ROC) curve analysis was applied. Following this, the cut-off value of SWE for discrimination be-

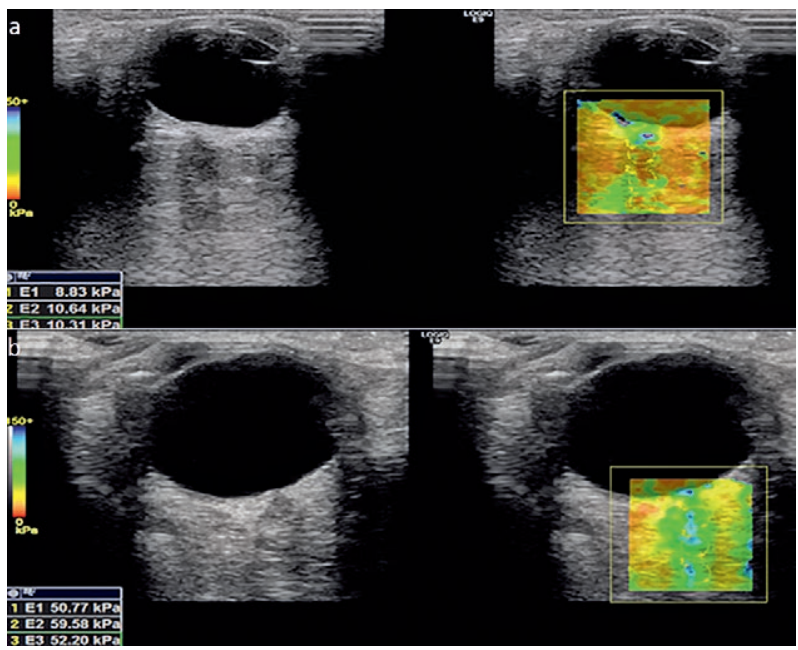

Fig 2. Elasticity measurement with shear-wave elastography on the optic nerve: a) of a 40-year-old woman, a healthy volunteer, showing a mean score of $9.92 \mathrm{kPa}$; b) of a 39-year-old man with multiple sclerosis, showing a mean score of $54.1 \mathrm{kPa}$. tween the groups was determined, and ROC curves were employed to describe the diagnostic sensitivity, specificity, PPV, and NPV

\section{Results}

In this study, we examined 54 MS patients (17 men; $38.87 \pm 10.5$ years; range, $17-59$ years), and 59 healthy volunteers (25 men; 38.31 \pm 11.6 years; range, 20-57 years). Both groups were compatible for age and gen$\operatorname{der}(\mathrm{p}=0.261, \mathrm{p}=0.289$, respectively). There were $14 \mathrm{MS}$ patients with previous optic neuritis (6 men; $38.7 \pm 10.3$ years; range, 22-59 years) and $40 \mathrm{MS}$ patients without previous optic neuritis ( $11 \mathrm{men} ; 38.9 \pm 10.8$ years; range, 17-56 years). These groups were also compatible for age and gender ( $\mathrm{p}=0.950, \mathrm{p}=0.296$, respectively).

The average duration of the disease was $7.56 \pm 5.83$ (range, 1-24) years in all MS patients. Mean optic nerve sheath diameter (ONSD) was $5.31 \pm 0.48 \mathrm{~mm}$ (range, 3.8-6.5) in all MS patients, $5.32 \pm 0.39 \mathrm{~mm}$ (range, 4.7$6.1)$ in MS with previous optic neuritis, $5.31 \pm 0.51 \mathrm{~mm}$ (range, 3.8-6.5) in MS without previous optic neuritis, and $5.22 \pm 0.58 \mathrm{~mm}$ (range, 3.7-7) in the healthy volunteers. There was no statistically significant difference in the mean thickness of the optic nerve between the MS patients with and without previous optic neuritis $(\mathrm{p}=0.920)$, and there was no statistically significant difference in the mean thickness of the optic nerve between all MS patients and healthy volunteers $(\mathrm{p}=0.205)$.

In the MS group, both eyes of one patient were excluded from the study because of glaucoma, and one patient's left eye was excluded because of retinal detachment surgery. One hundred and seven optic nerves were investigated among the MS patients, while 118 optic nerves were investigated in the healthy volunteers. Thus, a total of 225 optic nerves were prospectively examined with SE and SWE, and these were evaluated by comparing the results of all three groups. There were 27 (25.2\%) eyes with optic neuritis in the MS group, while there were $80(74.8 \%)$ eyes without optic neuritis.

The elasticity types and SWE values of optic nerves are summarized in Table I.

There was no significant correlation between MS disease duration and SWE values for all MS patients $(p=0.421)$, and there was not significant correlation between SWE values and optic nerve thickness or age in the whole MS patient group $(\mathrm{p}=0.221, \mathrm{p}=0.643)$. Similarly, there was no significant correlation between SWE values and optic nerve thickness or age in the healthy volunteers $(\mathrm{p}=0.505, \mathrm{p}=0.782$, respectively).

ROC curve analysis gave a value of $0.993(\mathrm{p}<0.001$; $95 \%$ CI $=0.971-0.999)$. The shear value of $18.3 \mathrm{kPa}$ had 
Table I. Elasticity types in strain elastography and shear wave elastography values of optic nerves in multiple sclerosis and healthy volunteers.

\begin{tabular}{|c|c|c|c|c|c|c|}
\hline \multirow[t]{2}{*}{ Group } & \multicolumn{4}{|c|}{ Elasticity types (SE) } & \multicolumn{2}{|l|}{ SWE } \\
\hline & Type 1 & Type 2 & Type 3 & p values & values $(\mathrm{kPa})$ & p values \\
\hline MS with ON (n=27) & $3(10.7 \%)$ & $22(78.6 \%)$ & $2(7.1 \%)$ & & $33.87 \pm 11.6($ range, $13-71.5)$ & \\
\hline MS without $\mathrm{ON}(\mathrm{n}=80)$ & $2(2.5 \%)$ & $73(91.7 \%)$ & $5(6.2 \%)$ & & $33.88 \pm 11.33$ (range, $16-55.3)$ & \\
\hline MS totally $(n=107)$ & $5(4.6 \%)$ & $95(88 \%)$ & $7(6.5 \%)$ & $(n<0,001)$ & $33.87 \pm 11.64$ (range, 13-71.5) & $(n<0,00$ \\
\hline Healthy volunteers $(\mathrm{n}=118)$ & $0(0 \%)$ & $45(38.1 \%)$ & $73(61.9 \%)$ & $(p<0.001)$ & $10.381 \pm 3.48$ (range, $4.8-22$ ) & $(p<0.001)$ \\
\hline
\end{tabular}

$\mathrm{n}$ - number of optic nerves, MS - multiple sclerosis, ON - optic nerve, SE - strain elastography, SWE - shear wave elastography

a very high sensitivity and specificity $(95.3-97.5 \%)$ for the patient group, and by the use of ROC curve analysis, it was determined that the best cut off value for MS was $18.3 \mathrm{kPa}$, which had a NPV of $95.8 \%$ and a PPV of $97.19 \%$.

\section{Discussions}

In this prospective study, we assessed the clinical usefulness of SE and SWE in evaluating the optic nerve involvement in MS patients in comparison to healthy volunteers. The results of this study showed that optic nerve stiffness evaluated by SE and SWE is significantly increased in patients with MS compared to healthy subjects but no significant differences were found between patient with and without previous optic neuritis. Accordingly, it may be presumed that optic nerve involvement in MS is associated with considerable hardening of the nerve tissue. Our study represents the first elastographic investigation of the optic nerve in patients with MS using SE and SWE techniques, as described above.

Optic neuritis is widespread localized inflammatory demyelination; it is an immune-mediated inflammatory condition of the optic nerve. Optic neuritis is typically seen in younger patients, often as the initial presenting feature of the disease. Acute visual loss is frequently a conclusion of acute demyelination of the optic nerve, and almost one half of MS patients are exposed to it at some point in the course of their disease. Vision loss substantially recovers after resolution of inflammation, but a residual vision deficit may persist. Histopathological research of the optic nerve in MS showed that prominent axonal loss occurs and the optic nerve cross-sectional area decreases after optic neuritis [6,20,21]. In recent studies significant correlations between the histopathological aspect and the tissue stiffness determined by elastography were established [12,13,22,23].

MS is an autoimmune disease that primarily includes the white matter and is thought to be mediated by autoreactive lymphocytes that spread over the central nervous system, pathological changes involving oligodendrocyte, axonal and neuronal injury and glial scarring; these changes cause demyelination, neuronal death, and brain atrophy $[4,8,24]$. The visual pathways are commonly involved in MS pathology, with the optic nerve particularly sensitive to optic neuritis; optic nerve axons that survive the acute insult remain largely demyelinated within the lesion. In addition, it is believed that constant demyelination can make axons more undefended against damage and may potentially cause axonal disruption $[9,20]$. A fibrotic scar can be described as a mark left by the healing of the injured tissue as the consequence of failed tissue regeneration in neurological disorders [25]. Mohan et al suggested that the perivascular collagen network may have anti-inflammatory features that participate in restricting the spreading of MS lesions; they demonstrated widespread network of perivascular fibrosis in MS lesions accumulating around veins and venules [26,27]. The demyelination, remyelination, axonal degeneration in the optic nerve, and especially the perivascular fibrosis that occur in the optic nerve in MS disease are probably the causes for the optic nerve hardening. The rigid elasticity patterns in patients with MS without previous neuritis may be an early warning signs of optic nerve involvement. This situation can be monitored using sonoelastography. The results of our study, by demonstrating the changes in tissue elasticity of the optic nerves in MS patients using SE and SWE, offer an alternative, a noninvasive diagnostic method for determining optic nerve involvement in these patients.

Ultrasound is one of the safest known radiological methods, and no significant side effects were reported until today. Also, ocular ultrasound, including Doppler ultrasound is recognized as being a safe, easy to perform method, with no ionizing radiation, that can be used as an imaging method in patients with MS disease and optic neuritis $[21,28]$. Despite the concerning for the possible biological and side effects of the ocular elastography, there are studies that reported the techniques to be safe and rapid for evaluating the retrobulbar fat tissue and the optic nerve [12,29-31].

This research had some limitations. First, SE is a highly operator dependent method, and strain values may vary on the degree of compression, as well as with the 
composition and structure of the tissues. Secondly, we appreciated the types of elasticity pattern qualitatively, by visual analysis of the color map, this being a subjective method. Therefore, to reduce the effect of the subjective evaluation, we combined SE with the SWE elastography procedure, which is quantitative and less operator dependent. Finally, we did not evaluate the interobserver variability in this study.

In conclusion, our prospective study demonstrated that SE and SWE may be useful for optic nerve investigations. The results of SE and SWE elastography were significantly meaningful in MS patients according to healthy volunteers. Therefore, both methods may be helpful in the early diagnosis of inflammatory diseases involving optic nerve, as well as the investigation of optic nerve and optic neuritis in MS patients. Both methods have some advantages over other approaches; for instance, they are low-cost imaging modalities, they do not include ionizing radiation, they do not require contrast agents and the methods are non-invasive. Nevertheless, it is obvious that there is need for more research corroborating the diagnostic performance of SE and SWE in relation to the optic nerve.

\section{Conflict of interest: none}

\section{References}

1. Raz E, Bester M, Sigmund EE, et al. A better characterization of spinal cord damage in multiple sclerosis: a diffusional kurtosis imaging study. AJNR Am J Neuroradiol 2013;34:1846-1852.

2. Hojjat SP, Kincal M, Vitorino R, et al. Cortical Perfusion Alteration in Normal-Appearing Gray Matter Is Most Sensitive to Disease Progression in Relapsing-Remitting Multiple Sclerosis. AJNR Am J Neuroradiol 2016;37:1454-1461.

3. Chawla S, Kister I, Wuerfel J, et al. Iron and Non-Iron-Related Characteristics of Multiple Sclerosis and Neuromyelitis Optica Lesions at 7T MRI. AJNR Am J Neuroradiol 2016;37:1223-1230

4. Assaf Y, Chapman J, Ben-Bashat D, et al. White matter changes in multiple sclerosis: correlation of q-space diffusion MRI and 1H MRS. Magn Reson Imaging 2005;23:703710 .

5. Khanna S, Sharma A, Huecker J, Gordon M, Naismith RT, Van Stavern GP. Magnetic resonance imaging of optic neuritis in patients with neuromyelitis optica versus multiple sclerosis. J Neuroophthalmol 2012;32:216-220.

6. Kolbe S, Chapman C, Nguyen T, et al. Optic nerve diffusion changes and atrophy jointly predict visual dysfunction after optic neuritis. Neuroimage 2009;45:679-686.

7. Inal M, Unal B, Kala I, Turkel Y, Bilgili YK. ADC evaluation of the corticospinal tract in multiple sclerosis. Acta Neurol Belg 2015;115:105-109.
8. Ge Y. Multiple sclerosis: the role of MR imaging. AJNR Am J Neuroradiol 2006;27:1165-1176.

9. Trip SA, Wheeler-Kingshott C, Jones SJ, et al. Optic nerve diffusion tensor imaging in optic neuritis. Neuroimage 2006;30:498-505.

10. Sbardella E, Tona F, Petsas N, Pantano P. DTI Measurements in Multiple Sclerosis: Evaluation of Brain Damage and Clinical Implications. Mult Scler Int 2013;2013:671730.

11. Anik Y, Demirci A, Efendi H, Bulut SS, Celebi I, Komsuoglu S. Evaluation of normal appearing white matter in multiple sclerosis: comparison of diffusion magnetic resonance, magnetization transfer imaging and multivoxel magnetic resonance spectroscopy findings with expanded disability status scale. Clin Neuroradiol 2011;21:207-215.

12. Vural M, Acar D, Toprak U, et al. The evaluation of the retrobulbar orbital fat tissue and optic nerve with strain ratio elastography. Med Ultrason 2015;17:45-48.

13. Thitaikumar A, Ophir J. Effect of lesion boundary conditions on axial strain elastograms: a parametric study. Ultrasound Med Biol 2007;33:1463-1467.

14. Tan S, Özcan MF, Tezcan F, et al. Real-time elastography for distinguishing angiomyolipoma from renal cell carcinoma: preliminary observations. AJR Am J Roentgenol. 2013;200:W369-W375.

15. Detorakis ET, Drakonaki EE, Tsilimbaris MK, Pallikaris IG, Giarmenitis S. Real-time ultrasound elastographic imaging of ocular and periocular tissues: a feasibility study. Ophthalmic Surg Lasers Imaging 2010;41:135-141.

16. Teber MA, Tan S, Dönmez U, et al. The use of real-time elastography in the assessment of gallbladder polyps: preliminaryobservations. Med Ultrason 2014;16:304-308.

17. Polman CH, Reingold SC, Banwell B, et al. Diagnostic criteria for multiple sclerosis: 2010 revisions to the McDonald criteria. Ann Neurol 2011;69:292-302.

18. Ballantyne SA, O’Neill G, Hamilton R, Hollman AS. Observer variation in the sonographic measurement of optic nerve sheath diameter in normal adults. Eur J Ultrasound 2002;15:145-149.

19. Ueda T, Ishida E, Kojima Y, Yoshikawa S, Yonemoto H. Sonographic Optic Nerve Sheath Diameter: A Simple and Rapid Tool to Assess the Neurologic Prognosis After Cardiac Arrest. J Neuroimaging 2015;25:927-930.

20. Graham SL, Klistorner A. Afferent visual pathways in multiple sclerosis: a review. Clin Exp Ophthalmol 2016 Mar 24. doi: 10.1111/ceo.12751.

21. Akçam HT, Capraz IY, Aktas Z, et al. Multiple sclerosis and optic nerve: an analysis of retinal nerve fiber layer thickness and color Doppler imaging parameters. Eye (Lond) 2014;28:1206-1211.

22. Garra BS. Imaging and estimation of tissue elasticity by ultrasound. Ultrasound Q 2007;23:255-268.

23. Chang JM, Moon WK, Cho N, Kim SJ. Breast mass evaluation: factors influencing the quality of US elastography. Radiology 2011;259:59-64.

24. Carrai V, Donnini I, Mazzanti B, et al. Immunohistochemistry analysis of bone marrow biopsies in multiple sclerosis patients undergoing autologous haematopoietic stem cells 
transplantation. Clin Neurol Neurosurg 2013;115:10441048.

25. Fernández-Klett F, Priller J. The fibrotic scar in neurological disorders. Brain Pathol 2014;24:404-413.

26. Mohan H, Krumbholz M, Sharma R, et al. Extracellular matrix in multiple sclerosis lesions: Fibrillar collagens, biglycan and decorin are upregulated and associated with infiltrating immune cells. Brain Pathol 2010;20:966-975.

27. Brown WR, Thore CR. Perivascular fibrosis in multiple sclerosis lesions. Brain Pathol 2011;21:355.

28. Bedi DG, Gombos DS, Ng CS, Singh S. Sonography of the eye. AJR Am J Roentgenol 2006;187:1061-1072.
29. Chang JM, Won JK, Lee KB, Park IA, Yi A, Moon WK. Comparison of shear-wave and strain ultrasound elastography in the differentiation of benign and malignant breast lesions. AJR Am J Roentgenol 2013;201:W347W356.

30. 30. Pekel G, Ağladioğlu K, Acer S, Bozkurt K, Çetin EN, Yağc1 R. Evaluation of ocular elasticity in high myopia. Optom Vis Sci 2015;92:573-578.

31. 31. Pekel G, Ağladığlu K, Acer S, Yağcı R, Kaşıkçı A. Evaluation of ocular and periocular elasticity after panretinal photocoagulation: an ultrasonic elastography study. Curr Eye Res 2015;40:332-337. 